\title{
Perceptions of Readmitted Patients on the Transition From Hospital to Home
}

\author{
Shreya Kangovi, MD ${ }^{1,2,4 *}$, David Grande, MD, MPA'3,4, Patricia Meehan, LSW5, Nandita Mitra, PhD', \\ Richard Shannon, $\mathrm{MD}^{3}$, Judith A. Long, $\mathrm{MD}^{1,3,4,7}$
}

\begin{abstract}
${ }^{1}$ Philadelphia Veterans Affairs Medical Center, Philadelphia, Pennsylvania; ${ }^{2}$ Robert Wood Johnson Foundation Clinical Scholars Program, University of Pennsylvania, Philadelphia, Pennsylvania; ${ }^{3}$ Department of Medicine, University of Pennsylvania Perelman School of Medicine, Philadelphia, Pennsylvania; ${ }^{4}$ Leonard Davis Institute of Health Economics, University of Pennsylvania, Philadelphia, Pennsylvania; ${ }^{5}$ Department of Social Work, University of Pennsylvania Health System, Philadelphia, Pennsylvania; ${ }^{6}$ Department of Biostatistics and Epidemiology, University of Pennsylvania, Philadelphia, Pennsylvania; 'Philadelphia VA Center for Health Equities Research and Promotion, Philadelphia, Pennsylvania.
\end{abstract}

BACKGROUND: Hospital leaders have had mixed success reducing readmissions Little is known about the readmitted patient's perspective.

METHODS: A cross-sectional 36-item survey was administered to 1084 readmitted inpatients of The Hospital of the University of Pennsylvania (an urban academic medical center) and Penn Presbyterian Medical Center (an urban community hospital) between November 10, 2010 and July 5, 2011. The survey response rate was $32.9 \%$.

RESULTS: The most commonly reported issues contributing to readmission were: 1) feeling unprepared for discharge (11.8\%); 2) difficulty performing activities of daily living (ADLs) (10.6\%); 3) trouble adhering to discharge medications (5.7\%); 4) difficulty accessing discharge medications (5.0\%); and 5) lack of social support (4.7\%). Low-socioeconomic status (SES) (defined as uninsured or Medicaid) patients were more likely than high-SES patients to report difficulty understanding (odds ratio [OR] 2.7; 95\% confidence interval [Cl] 1.1, 6.6) and executing (OR 2.2; 95\% Cl 1.1, 4.4) discharge instructions, difficulty adhering to medications (OR 1.8; $95 \% \mathrm{Cl} 1.2,3.0$ ), lack of social support (OR 2.0; $95 \% \mathrm{Cl}$ $1.2,3.6)$, lack of basic resources (OR 2.6; $95 \% \mathrm{Cl} 1.1,6.1)$, and substance abuse (OR 6.7; 95\% Cl 2.3, 19.2).

CONCLUSIONS: Patients reported transition challenges which they believe contribute to illness relapse and readmission. Interventions designed to address these challenges, and tailored for patient characteristics such as SES, may better address the root causes of readmission. Journal of Hospital Medicine 2012;7:709-712. (C) 2012 Society of Hospital Medicine
Over $14 \%$ of all patients hospitalized in the United States are readmitted within 30 days of discharge. ${ }^{1}$ Numerous studies have used administrative data in order to identify clinical and operational predictors of readmission. However, few studies have explored patients' perspectives on readmission..$^{2-7}$ As a result, we know little about potentially modifiable challenges which patients face during the transition from hospital to home. Lack of understanding of the patient perspective has hampered the ability of hospitals to create interventions which address these underlying causes of readmissions.

Patients with low socioeconomic status (SES) are up to $43 \%$ more likely to require readmission than their higher-SES counterparts, ${ }^{8,9}$ and qualitative data has described unique challenges faced by low-SES patients during transition. ${ }^{2}$ Our objectives were to understand the transition experiences of readmitted patients and

\footnotetext{
*Address for correspondence and reprint requests: Shreya Kangovi, MD, University of Pennsylvania, 423 Guardian Dr, 13th Floor, Blockley Hall, Philadelphia, PA 19104; Telephone: 215-520-5309; Fax: 215-573-2742; E-mail: kangovi@mail.med.upenn.edu

Additional Supporting Information may be found in the online version of this article.

Received: February 27, 2012; Revised: June 20, 2012; Accepted: July 5,2012

2012 Society of Hospital Medicine DOI 10.1002/jhm.1966

Published online in Wiley Online Library (Wileyonlinelibrary.com).
}

to compare these experiences across SES and diagnostic categories.

\section{METHODS}

\section{Development of a Survey Instrument}

A collaborative team of physicians, nurses, and social workers used a previously defined conceptual framework, ${ }^{10}$ literature search, and expert interviews to construct a 36-item survey that addressed the following domains: preparedness for prior discharge; delays in care-seeking; medication adherence; follow-up with a primary care provider (PCP); and overarching challenges faced during transition which contributed to readmission. Each question had multiple answer choices including "Other" which allowed patients to provide open-ended answers; patients could select all answer choices that applied. Prior to administration, the survey was pretested with 15 random patients and revised to improve reliability and comprehensibility. (See Supporting Information, Survey Script Versions 1.0 and 2.0, in the online version of this article.)

\section{Sampling Strategy and Patient Enrollment}

Patients were eligible to participate if they: 1) had capacity to complete an interview; and 2) were readmitted within 30 days of a prior discharge from the Hospital of the University of Pennsylvania (HUP), a 695-bed academic medical center, or Penn Presbyterian 
TABLE 1. Characteristics of Surveyed Patients vs. Non-surveyed

\begin{tabular}{|c|c|c|c|}
\hline Characteristics of Patients & $\begin{array}{l}\text { Survey Sample } \\
\qquad(n=1084)\end{array}$ & $\begin{array}{l}\text { Not in Survey } \\
\text { Sample } \\
(\mathrm{n}=2797)\end{array}$ & $P$ Value $^{*}$ \\
\hline $\begin{array}{l}\text { Age mean (SD) } \\
\text { Gender } n(\%)\end{array}$ & $55.7(16.6)$ & $58.0(18.2)$ & $<0.01$ \\
\hline $\begin{array}{l}\text { Gender, n }(\%) \\
\text { Male }\end{array}$ & $546(50.4 \%)$ & $1428(51.1 \%)$ & 0.88 \\
\hline Race, $n(\%)$ & & & 0.96 \\
\hline Black & $502(46.4 \%)$ & $1146(41.3 \%)$ & \\
\hline White & $504(46.6 \%)$ & $1362(49.1 \%)$ & \\
\hline $\begin{array}{l}\text { Principal discharge diagnosis, } \uparrow n(\%) \\
\text { Medical }\end{array}$ & & & 0.98 \\
\hline $\begin{array}{l}\text { Acute on chronic systolic } \\
\text { heart failure }\end{array}$ & $44(4.6 \%)$ & $23(1.3 \%)$ & \\
\hline Acute renal failure & $24(2.5 \%)$ & $29(1.7 \%)$ & \\
\hline Surgical & & & \\
\hline Postoperative infection & $48(14.8 \%)$ & $53(5.2 \%)$ & \\
\hline Digestive system problems & $17(5.2 \%)$ & $23(2.2 \%)$ & \\
\hline APR-DRG score, $n(\%)$ & & & 0.13 \\
\hline 0 (Not assigned) & $9(0.7 \%)$ & $28(1.0 \%)$ & \\
\hline 1 (Minor) & $113(10.1 \%)$ & $628(22.7 \%)$ & \\
\hline 2 (Moderate) & $338(31.4 \%)$ & $881(31.8 \%)$ & \\
\hline 3 (Major) & $470(43.7 \%)$ & $883(31.9 \%)$ & \\
\hline 4 (Extreme) & $154(14.3 \%)$ & $369(13.3 \%)$ & \\
\hline Length of stay mean (SD) & $6.2(6.9)$ & $6.5(10.1)$ & 0.33 \\
\hline Insurance payer, n (\%) & & & 0.77 \\
\hline Uninsured/Medicaid & $234(21.6 \%)$ & $489(17.5 \%)$ & \\
\hline Medicaid + Medicare & $85(7.84 \%)$ & $172(6.2 \%)$ & \\
\hline Medicare & $345(31.8 \%)$ & $878(31.5 \%)$ & \\
\hline Private & $420(38.8 \%)$ & $1253(44.9 \%)$ & \\
\hline No. of 60-d readmissions mean (SD) & $1.3(0.02)$ & $2.0(0.02)$ & $<0.01$ \\
\hline
\end{tabular}

Abbreviations: APR-DRG, All Patient Refined-Diagnosis Related Groups; SD, standard deviation. ${ }^{*} t$ test for continuous variables and $\chi^{2}$ for categorical variables. International Classification of Diseases, Ninth Revision (ICD-9) codes for conditions listed within medical and surgical groups are as follows: acute on chronic systolic heart failure (428.23), acute renal failure (584.9), postoperative infection (998.59), digestive system problems (997.4)

Medical Center (PPMC), a 317-bed affiliated community hospital. Both hospitals are located in Philadelphia and serve a population which is $45.4 \%$ privately insured, $33.5 \%$ insured by Medicare, and $21.2 \%$ uninsured or insured by Medicaid. We excluded readmissions that were planned or from another facility because these were less sensitive to patient domains such as adherence, access, and social support.

Eligible participants were identified by survey administrators (bedside nurses, social workers, or clinical resource managers) on the day of hospital readmission. Because data were being used immediately for quality improvement, the Institutional Review Board (IRB) waived the need for consent. Administrators typically took 10 minutes to conduct the survey in-person and record responses directly into patients' electronic medical record (EMR). Inpatient care teams could view responses in real time and work to resolve identified challenges prior to patients' discharge.

Between November 10, 2010 and July 5, 2011, 3881 patients were readmitted to study hospitals. Five hundred eighty-four readmissions were ineligible for the study because they lacked capacity, were planned readmissions, or were readmitted from another facility. This left 3297 eligible individuals. We surveyed
1084 individuals yielding a response rate of $32.9 \%^{11}$; the remainder either refused the survey, or were not approached for the survey due to time restraints of administrators. Characteristics of responders and nonresponders are displayed in Table 1, and were similar in all measured categories with the exception of age (58.0 vs $55.7, P<0.01$ ) and the number of 60 -day readmissions ( 2.0 vs $1.3, P<0.01)$.

\section{Statistical Analysis}

Survey responses were extracted from the EMR and linked with patient clinical and demographic data. Variables pertaining to hospitalization, such as admitting service and principal diagnosis, were associated with patients' index hospitalization rather than the readmission. A trained research assistant extracted open-ended free-text answers to any survey questions marked, "Other" and coded them using a grounded theory approach. ${ }^{12}$

In our primary analysis, we described challenges reported by readmitted patients. In a secondary analysis, we tested for differences in transition challenges by SES using lack of insurance or Medicaid as a proxy for low SES. Using insurance status as a marker for material aspects of SES is well-described in health services research. ${ }^{13-16}$ In addition, income data from our institution demonstrated that $86.5 \%$ of uninsured and Medicaid patients have a median household income below $\$ 15,000$. We tested for differences by diagnostic category using the index admitting service (medical vs surgical) as a proxy for diagnostic category (Table 2).

We compared continuous variables using the two-sample $t$ test and categorical variables using Pearson's chi-square test. The Cuzick nonparametric test was used to test for trends across ordered groups. We used multivariable logistic regression models to estimate the association between each binary transition challenge outcome and predictors: SES and diagnostic group. These models were adjusted for potential confounders: age, gender, length of stay, and severity of illness, as determined by All Patient Refined-Diagnosis

TABLE 2. Multivariable Logistic Regression Models of Transition Challenges

\begin{tabular}{lcc}
\hline & $\begin{array}{c}\text { Low vs High } \\
\text { SES (ref) OR } \\
(95 \% \text { Cl) }\end{array}$ & $\begin{array}{c}\text { Medical vs } \\
\text { Surgical (ref) } \\
\text { OR }(95 \% \mathrm{Cl})\end{array}$ \\
\hline Unprepared for DC & $1.3(0.9,1.9)$ & $1.0(0.7,1.6)$ \\
Understanding DC instructions & $2.7(1.1,6.6)$ & $1.7(0.5,5.8)$ \\
Executing DC instructions & $2.2(1.1,4.4)$ & $1.6(0.6,3.7)$ \\
Activities of daily living & $1.0(0.6,1.5)$ & $1.1(0.7,1.7)$ \\
Medication access & $1.6(0.9,2.8)$ & $2.3(1.0,4.9)$ \\
Medication adherence & $1.8(1.2,3.0)$ & $2.6(1.2,5.4)$ \\
Lack of social support & $2.0(1.2,3.6)$ & $2.3(1.0,5.2)$ \\
Lack of food, transportation, telephone & $2.6(1.1,6.1)$ & $7.1(0.9,53.2)$ \\
Substance abuse & $6.7(2.3,19.2)$ & $1.5(0.4,5.2)$ \\
\hline
\end{tabular}

Abbreviations: $\mathrm{Cl}$, confidence interval; $\mathrm{DC}$, discharge; OR, odds ratio; ref, reference; SES, socioeconomic status. 
Related Groups (APR-DRGs). We did not adjust for race because it was strongly correlated with SES in our dataset $(P<0.0001)$. Confounders were included in final models if their association with outcomes had a $P$ value less than 0.10. Analyses were performed using the STATA software package, version 11.0 (StataCorp LP, College Station, TX). The survey was approved by the University of Pennsylvania IRB.

\section{RESULTS}

\section{Patient Characteristics}

We surveyed 1084 unique individuals; $50.4 \%$ of participants were male, $46.4 \%$ were black. The most common index principal diagnosis in the medical group was systolic heart failure $(4.6 \%)$, while the most common index principal diagnosis in the surgical group was postoperative infection (14.8\%) (Table 1$)$.

\section{Discharge Preparedness, Medication Adherence, and PCP Follow-up}

At the time of prior discharge, $86.4 \%$ of respondents felt that they had been prepared for self-care. $80.3 \%$ reported being able to take all discharge medications as prescribed. The most common reasons for not being able to take medications included: 1) side effects or worry about side effects $(13.1 \%) ; 2$ ) trouble paying for medications $(10.7 \%)$; and 3$)$ lack of transportation to the pharmacy $(8.4 \%)$. Since their prior discharge, $52.9 \%$ of participants reported that they had visited a PCP; $28.7 \%$ of participants report being referred by their PCP to the emergency room for readmission.

\section{Transition Challenges in Overall Survey Sample}

During the transition from hospital to home, $45.5 \%$ of readmitted patients reported experiencing challenges which contributed to readmission. The most commonly reported issues contributing to readmission were: 1) feeling unprepared for discharge $(11.8 \%)$; 2 ) difficulty performing activities of daily living (ADLs) $(10.6 \%) ; 3)$ trouble adhering to discharge medications $(5.7 \%) ; 4)$ difficulty accessing discharge medications $(5.0 \%)$; and 5$)$ lack of social support $(4.7 \%)$.

\section{Transition Challenges by Subgroup}

Low-SES patients were more likely than high-SES patients to report difficulty understanding (odds ratio [OR] 2.7; 95\% confidence interval $[\mathrm{CI}] 1.1,6.6)$ and executing (OR 2.2; 95\% CI 1.1, 4.4) discharge instructions, difficulty adhering to medications (OR $1.8 ; 95 \%$ CI 1.2, 3.0), lack of social support (OR 2.0; $95 \%$ CI 1.2, 3.6), lack of basic resources (OR 2.6; $95 \%$ CI 1.1, 6.1), and substance abuse (OR 6.7; 95\% CI 2.3, 19.2) as perceived reasons for readmission. Of the patients who described "Other" issues contributing to readmission, low-SES patients most commonly described stress or depression $(49.0 \%)$, while highSES patients most commonly reported a recurrence of symptoms (74.8\%). Medical and surgical patients had similar odds of facing each transition challenge with one exception: medical patients were more likely to report difficulty adhering to medications (OR 2.6; $95 \%$ CI 1.2, 5.4).

\section{DISCUSSION}

Several findings from this study are of interest to practicing hospitalists or hospital administrators. First, of the issues to which patients most commonly attributed readmission, lack of discharge preparedness is the only one which occurs during index hospitalization; in order to address most transition challenges, hospitals must think beyond their walls. By penalizing hospitals for excess rates of readmission, The Hospital Readmission Reduction Program (HRRP) will effectively hold hospitals accountable for addressing issues which occur in patients' homes and communities. ${ }^{17}$ Hospitals that have robust partnerships with community pharmacies, social service agencies, and PCPs may have the most influence on these issues and the most success in reducing readmissions. Second, consistent with other literature describing increased rates of readmission with enhanced PCP follow-up, ${ }^{18}$ our findings demonstrate that PCPs often refer their patients to the emergency room for readmission. This suggests that PCP follow-up, while perhaps essential for patient care, may not necessarily reduce readmissions and may actually facilitate readmission. Third, this study describes underlying reasons for patient nonadherence with discharge medications: side effects, cost, and transportation. Targeted interventions to improve adherence may include floor-based pharmacists who counsel on side effects, determine co-pays prior to discharge, and encourage patients to fill prescriptions from the hospital pharmacy to avoid transportation barriers.

Finally, and perhaps most importantly, these data suggest that one transition experience does not fit all. Patients with low SES appear to have a distinct and challenging transition experience. Currently, there is an emphasis on tailoring transition interventions to specific disease populations, such as patients with congestive heart failure. Our study suggests that it may be more effective to tailor interventions for low-SES patients across diagnostic category, helping these patients gain access to outpatient medical resources and address competing issues, such as food insecurity or substance abuse.

Our study has several limitations. First, the low survey response rate makes it susceptible to nonresponse bias. Second, survey administration by a member of the care team may have increased social desirability bias. Third, because it was important to the study team to incorporate our survey into hospital workflow, survey responses were recorded directly into the EMR which limited administrators to recording a "yes" response for each answer choice which the participant endorsed. Therefore, in our dataset, we are unable to distinguish a definite "no" from a missing response; however, the survey was short, making it 
unlikely that questions were skipped. Fourth, closedended questions may have failed to capture the range of participant responses, although the inclusion of an open-ended answer choice ameliorates this issue. Finally, we are unable to draw conclusions regarding association of survey responses with the risk of readmission, because this study was administered only to readmitted patients.

\section{CONCLUSIONS}

This report of patients' perspectives challenges many commonly held assumptions regarding readmission. Readmission reflects not only the quality of hospital care, but a variety of factors in patients' homes and communities. PCP follow-up, while perhaps critical for patient care, may not be a panacea for reducing hospital readmissions. Targeted medication counseling focused on side effects, co-pay, and medication delivery may address patients' underlying reasons for nonadherence. And most importantly, one transition experience does not fit all. Hospitalists and administrators must tailor interventions to address challenges reported by their patients, particularly those of low SES.

\section{Acknowledgements}

The authors are grateful to the Society of General Internal Medicine (SGIM) for selecting our abstract "Perceptions of Readmitted Patients on the Transition From Hospital to Home" as a Lipkin Award Finalist during the 2012 SGIM National Meeting.

Disclosures: Support for this study was provided by a grant from the Leonard Davis Institute of Health Economics. Dr Grande has received honoraria from the Johns Hopkins University CME Program; has a consultancy with the National Nursing Centers Consortium; and has received grant support from, or has grants pending with, the HealthWell Foundation, the National Human Genome Research Institute, and the Agency for Healthcare Research and Quality. Dr Shannon is the founder of a biotech company, Ventrigen, LLC; is a senior fellow at IHI; is on the scientific advisory boards for Glasgow Smith Klein, Pfizer, Merck, and Value Capture; and is a member of the Board of Directors of the ABIM.

\section{References}

1. Wier LM, Barrett M, Steiner C, Jiang HJ. All-Cause Readmissions by Payer and Age, 2008: Statistical Brief \#115. Healthcare Cost and Utilization Project (HCUP) Statistical Briefs. Rockville, MD: Agency for Health Care Policy and Research; February 2006-June 2011.

2. Strunin L, Stone M, Jack B. Understanding rehospitalization risk: can hospital discharge be modified to reduce recurrent hospitalization? J Hosp Med. 2007;2(5):297-304.

3. Weaver F, Perloff L, Waters T. Patients' and caregivers' transition from hospital to home: needs and recommendations. Home Health Care Serv Q. 1999;17(3):27-48.

4. Annema C, Luttik ML, Jaarsma T. Reasons for readmission in heart failure: perspectives of patients, caregivers, cardiologists, and heart failure nurses. Heart Lung. 2009;38(5):427-434.

5. Fetter MS, Lowery BJ. Psychiatric rehospitalization of the severely mentally ill: patient and staff perspectives. Nurs Res. 1992;41(5): 301-305.

6. Soler RS, Juvinya Canal D, Noguer CB, Poch CG, Brugada Motge N, Del Mar Garcia Gil M. Continuity of care and monitoring pain after discharge: patient perspective. J Adv Nurs. 2010;66(1):40-48.

7. Procter S, Wilcockson J, Pearson P, Allgar V. Going home from hospital: the carer/patient dyad. J Adv Nurs. 2001;35(2):206-217.

8. Weissman JS, Stern RS, Epstein AM. The impact of patient socioeconomic status and other social factors on readmission. A prospective study in 4 Massachusetts hospitals. Inquiry. 1994;31(2):163-172.

9. Allaudeen N, Vidyarthi A, Maselli J, Auerbach A. Redefining readmission risk factors for general medicine patients. J Hosp Med. 2011; 6(2):54-60

10. Kangovi S, Grande D. Hospital readmissions-not just a measure of quality. JAMA. 2011;306(16):1796-1797.

11. American Association for Public Opinion Research (AAPOR). Standard Definitions: Final Dispositions of Case Codes and Outcome Rates for Surveys. 7th ed. Deerfield, IL: AAPOR; 2011.

12. Glaser BG, Strauss AL. The Discovery of Grounded Theory: Strategies for Qualitative Research. New York: Aldine; 1967.

13. Ayanian JZ, Kohler BA, Abe T, Epstein AM. The relation between health insurance coverage and clinical outcomes among women with breast cancer. N Engl J Med. 1993;329(5):326-331.

14. Foraker RE, Rose KM, Whitsel EA, Suchindran CM, Wood JL, Rosamond WD. Neighborhood socioeconomic status, Medicaid coverage and medical management of myocardial infarction: atherosclerosis risk in communities (ARIC) community surveillance. BMC Public Health. 2010;10:632.

15. Harnick DJ, Cohen JL, Schechter CB, Fuster V, Smith DA. Effects of practice setting on quality of lipid-lowering management in patients with coronary artery disease. Am J Cardiol. 1998;81(12): $1416-1420$.

16. Store PMD. Income Data by Insurance Category. 2012.

17. Kocher RP, Adashi EY. Hospital readmissions and the Affordable Care Act: paying for coordinated quality care. JAMA. 2011;306(16): $1794-1795$.

18. Weinberger M, Oddone EZ, Henderson WG. Does increased access to primary care reduce hospital readmissions? N Engl J Med. 1996; 334(22):1441-1447. 